DOI: 10.25100/eg.v0i21.11149

Espacios y Territorios

\title{
Desistimiento al delito mediante procesos de labor social en el jardín botánico de Cali, Colombia - Narrativas de acción y decisiones de la persona
}

\section{Desistance of Crime through social work processes in the botanical garden of Cali, Colombia - Action narratives and decisions of the person}

\author{
Alex Enrique Contreras Sánchez \\ Doctor (C) en Sociología. Universidad Nacional de Educación a Distancia. San Sebastián, España. \\ $\underline{\text { alexenriquecontreras@gmail.com | 0000-0002-8455-3192 }}$ \\ Isabel Elena Muñoz Galvis \\ Geógrafa. Universidad del Valle. Cali, Colombia

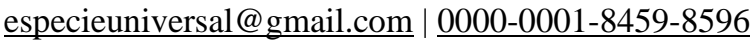

\begin{abstract}
Para citar este artículo: Contreras Sánchez, A. E., y Muñoz Galvis, I. E. (2021). Desistimiento al delito mediante procesos de labor social en el jardín botánico de Cali, Colombia - Narrativas de acción y decisiones de la persona. Entorno Geográfico, (21), 21-46. https://doi.org/10.25100/eg.v0i21.11149
\end{abstract}

\section{Resumen}

La desmovilización de grupos armados organizados al margen de la ley (GAOML), no es un precedente actual en Colombia, se ha realizado a lo largo del siglo XX, siendo la década de los 90 cuando la mayoría de los GAOML se han desmovilizado en Colombia. La importancia de este artículo de investigación resalta el punto de vista institucional a través de sus profesionales y la forma en que las Personas en Procesos de Reintegración (PPR) han desistido del delito y han vuelto a la vida civil. Estos procesos a través del trabajo social voluntario en el Jardín Botánico de Cali (JBC) entre los años 2016 a 2018, han permitido analizar: las rutas de desmovilización, el proceso realizado para volver a la vida civil y el trabajo desarrollado en parques, bosques y comunidades del municipio de Santiago de Cali, gracias al apoyo de la Secretaría de Paz y Cultura (SPC) de la Alcaldía de Cali y la Agencia Colombiana para la Reintegración (ACR), que durante el proceso pasó a denominarse Agencia para la Reincorporación y Normalización (ARN).

Palabras clave: Jardín Botánico Cali, Desistimiento, Desmovilización, Grupos Armados, Labor Social, Reintegración. 


\begin{abstract}
The demobilization of armed groups organized outside the law (GAOML), is not a current precedent in Colombia, it has been carried out throughout the 20th century, being the decade the 90s when most of the GAOML have demobilized in Colombia. The importance of this research article highlights the institutional point of view through its professionals and the way in which People in Reintegration Processes (PPR) have desisted from crime and returned to civil life. These processes through voluntary social work in the Botanical Garden from Cali (JBC) between the years 2016 to 2018, have allowed to analyze: the demobilization routes, the process carried out to return to civilian life and work developed in parks, forests and communities of the municipality of Santiago de Cali, thanks to the support of the Secretariat of Peace and Culture (SPC) of the Mayor's Office of Cali and the Colombian Agency for Reintegration (ACR), which during the process was renamed the Agency for Reincorporation and Normalization (ARN).
\end{abstract}

Keywords: Botanical Garden Cali, Desistance, Demobilization, Armed Groups, Reintegration

Recibido: 30 de noviembre de 2020

Aceptado: 24 de diciembre del 2020

\title{
Introducción
}

Tras la desmovilización de los grupos armados de izquierda más importantes en la década de los 90s: Movimiento 19 de Abril (M-19), Partido Revolucionario de los Trabajadores (PRT), Movimiento Armado Quintín Lame (MAQL), Ejército Popular de Liberación (EPL), Comando Ernesto Rojas (CER), Corriente de Renovación Socialista (CRS), Milicias Populares y el Frente Fernando Garnica (FFG) (Caicedo, 2016) \& (ARN, 2017), se inician los proceso de Desarme, Desmovilización y Reintegración (DDR) más importantes de nuestra contemporaneidad: a) Colectivos: Autodefensas Unidas de Colombia (AUC) en 2003 (Conpes 3554, 2008), Fuerzas Armadas Revolucionarias de Colombia -Ejercito del pueblo (FARCEP) en 2016 (Conpes 3931, 2018) y b) Individuales: Ejército de Liberación Nacional (ELN) y FRAC-EP (2000 a 2018) (ARN, 2020), teniendo en cuenta que entre los años 2019 y 2020, aún continúan las desmovilizaciones individuales del ELN, haciéndose necesario su desmovilización colectiva para llegar al fin estructural de la violencia política en Colombia de GAOML. 
En especial y en relación a nuestra temática, la desmovilización de la Corriente de Renovación Socialista (CRS) en 1994, sienta un precedente importante de procesos de acción con la creación del Jardín Botánico San Jorge en Tolima, a través de la Corporación Nuevo Arco Iris (SEMANA, 2009) por medio de un programa de desarrollo regional, en donde excombatientes desisten al delito, administrando y poniendo en marcha dicho jardín, junto a otras propuestas productivas.

Seguido de los procesos de Desarme, Desmovilización y Reintegración (DDR) de la década de los 90s, la transición en procesos DDR, en las dos últimas décadas, manifiesta dos modalidades de desmovilización: individuales y colectivas, siendo los grupos armados: AUC; ELN; FARC, los encargados de protagonizar dichos procesos. Ante este nuevo enfoque, el Jardín Botánico de Cali -JBC-, ha brindado la posibilidad a excombatientes de seguir con su proceso de desistimiento al delito por medio de Procesos de Labor Social (PLS) en sus instalaciones. Entendiendo aquí el desistimiento al delito como el proceso gradual, en que un sujeto abandona progresivamente la comisión de comportamientos antisociales (Laub y Sampson, 2001) o de retorno a la violencia, sustituyéndolos por nuevos patrones de carácter prosocial (Laub y Sampson, 2001; Maruna, 2001).

En relación con nuestro punto de partida, podemos decir que el JBC después de estar abierto una década (2001-2010), “cerró sus puertas entre los años 2010 a 2013” (E01), llevándole dos años para retomar sus labores. Fue solo a partir de 2015, que estableció su plan de investigaciones, centrándose en restauración ecológica y participación comunitaria. Dentro de sus proyecciones, se encontraba adelantar gestiones institucionales para ejecutar una intervención en el marco de la restauración ecológica para 2016. En ese sentido, se estableció una alianza con la Agencia Colombiana para la Reintegración (ACR), la Oficina de Paz de la Alcaldía de Santiago de Cali y el programa Hojas Verdes de Cotelvalle, para realizar un proceso de restauración ecológica del Bosque Seco Tropical, con la implementación de herramientas para el manejo del paisaje, donde se hizo intervención en un área de ladera de la ciudad, mediante la ejecución del servicio social de PPR a la vida civil (JBC, 2016a). El proyecto inició en 2016 y continuó hasta el 2018. En ese plazo, se involucraron "Juntas de Acción Comunal, Juntas de Acción Local y la Policía Nacional” (E02).

Gracias a los aportes en temas de paz por medio de la Red Nacional de Jardines Botánicos (RNJB), los directivos del JBC consideran que este jardín también es territorio de paz (E01, 
E02, E03 \& JB-UTP 2016), al estar rodeado por zonas rojas, "estamos en un área difícil, tenemos barrios vecinos que tienen conflictos, entonces hemos evaluado estas posibilidades y a su vez hemos considerado trabajar con estas comunidades" (E02), así pues, el JBC analiza factores de riesgo y busca factores de protección que beneficien el entorno.

Objetivo Exponer el punto de vista institucional del JBC y de las personas que, desmovilizadas, han hecho parte del proceso de labor social de la ARN y la SPC.

\section{Metodología}

El presente artículo forma parte del capítulo IV del Trabajo Fin de Master (TFM) presentado a la UOC (Contreras, 2018), coordinado por la Red Nacional de Jardines Botanicos de Colombia (RNJB) y el JBC. Se soporta a un estudio de perspectiva cualitativa para la cual se desarrolló una entrevista presentada a profesionales del JBC, una víctima y ha desmovilizados que han hecho parte del proceso de labor social de dicha institución, con la intención de "obtener informacion a través de las preguntas de un entrevistador y las respuesta de un entrevistado, normalmente experto en áreas del conocimeinto específico" (Cervera, 2019), para analizar narrativas de cambio que muestren como un excombatiente puede desistir al delito (procesos de acción o agencia), siendo estas la fuente fundamental de procedencia de datos con las que se ha trabajado.

El diseño está inspirado en un enfoque narrativo (Padrón, 2014) y un cuestionario semiestructurado (Achutegui, 2017), en el cual, para identificar las narrativas, se utilizó una simbología alfanumérica (Maruna, 2001; Achutegui, 2017), es decir: (1) La letra y (2) Número, con los cuales, se identificó el sujeto objeto de estudio, haciendo alusión al número de entrevista (E01: Entrevistado/a 01), el mismo que se utilizó para su identificación y el posterior análisis. El cuestionario contó con 35 preguntas y se dividió en 3 partes según las narrativas: Coordinadora (E01); Directores (E02, E03); Víctima (E04) y 4) PPR (E05, E06, E07, E08, E09). Cada uno de los entrevistados firmaron un documento de consentimiento informado, con la única salvedad de que los nombres de las PPR entrevistadas no aparecieran, sino con la categoría laboral por la que finalizaron su proceso de labor social. Cada entrevista fue realizada en las instalaciones del Jardín Botánico de Cali, logrando así, un análisis cualitativo de carácter narrativo y bibliográfico. Cada entrevista, permitió la identificación de dos criterios, (1) hacer una aproximación al PLS gestado en el JBC con Desmovilizados y (2) su criterio de selección, vinculación, labor y propuestas.

El instrumento de recolección de información fue la entrevista en profundidad, la cual, no se rigió esencialmente por criterios estadísticos y asumió intervalos de preguntas abiertas que 
permitieron a los entrevistados elaborar su propio discurso en relación a su historia de vida, con la intención de obtener, además de información, una visión autobiográfica, que siga las recomendaciones metodológicas de la tipología de la entrevista: (1) narrativa: trama clara, una interacción social y un despliegue temporal y (2) semi-estructurada: donde se obtenga descripciones del mundo de la vida del entrevistado con respecto a la interpretación del significado de los fenómenos descritos (Kvale, 2011).

Se optó por la alternativa exploratoria al existir un gran vacío bibliográfico sobre el tema y un espacio de literatura inexistente, no solo en Colombia sino a nivel internacional, al ser el objeto de estudio, desmovilizados con pena alternativa que no han perdido su libertad, presentándose como un punto de vista novedoso que representa un tema imprescindible al no existir documentación sobre este tema específico de atención. Al no encontrarse trabajos directamente relacionados con esta temática fue necesario para su desarrollo, acudir a investigaciones inéditas del JBC y a narrativas de vida de los desmovilizados, generándose así información empírica de primer orden.

\section{Resultados}

\section{Punto de vista institucional}

\section{Proceso 2016}

Este proceso inició el día 27 de abril de 2016, “fue diseñado en torno a una restauración ecológica" (E02), la cual se logró mediante una propuesta de servicio social a la ACR, denominada Herramientas de Paz para los Paisajes de mi Ciudad, buscando la restauración de una hectárea de Bosque Seco Tropical, mejor llamado Selva Seca Ecuatorial, puesto que "el bosque seco realmente es más de las tierras del norte" (E01). Tras este proyecto, prevalecía la idea de replicar dicho proceso en otras esferas de la vida, con la intención de ayudar a la construcción de una ciudadanía más conectada con la naturaleza (JBC, 2016b).

Su plan de trabajo se basó en la disponibilidad de horas de trabajo social por voluntariado, jornadas de preparación, actividades y talleres, para posteriormente, desarrollar actividades de campo. El personal del JBC se compuso por: Directora General, Coordinadora Educación, Jefe Colecciones, jardineros e intérpretes ambientales, que como equipo afianzaban la construcción del saber colectivo y procesos decisorios o de agencia, apostándole a los cambios y la transformación social, y considerando "que la organización del JBC es una plataforma para la formación ciudadana, el fortalecimiento de la identidad y la transformación 
de prácticas culturales" (E01). Lo único que se le solicitó a la ACR en ese momento fue "un asesor o persona encargada de realizar un acompañamiento general en todo el proceso" (E01).

La metodología empleada entre JBC y ACR en este periodo, fue Herramientas para el Manejo del Paisaje (HMP) (JBC, 2016c) que comprendió: control de invasoras, manejo y enriquecimiento del suelo, vivero, mantenimiento zona restauración, mantenimiento ribera río Cali y educación ambiental. Se contó con 27 PPR, de las cuales 5 eran mujeres, siendo la mayoría del pacífico colombiano y en menor medida de zonas urbanas: Buenaventura y Cali. Estas personas fueron las encargadas de la restauración de una hectárea de bosque sobre la ribera de la cuenca media del rio Cali. En cuanto al criterio de selección, el JBC aceptó dos modalidades de la ACR, "gente que tiene que cumplir sus ochenta horas de labor social y la que tiene que cumplir menos de ochenta" (E02). El tiempo utilizado para este primer proyecto fue de 4 meses.

Del proceso se pudo observar cómo se eliminó todo el pasto elefante, se limpió la zona, se sembraron 1000 árboles (38 especies nativas), se permitió todo aporte creativo y conocimiento activo obtenido de las labores del campo, realizando al final un picnic para la entrega del proyecto. Esta experiencia mostró cómo distintos sectores sociales pueden articularse y desarrollar iniciativas con impactos sociales y ecológicos positivos articulados a procesos de importancia nacional. La entrega, compromiso y apropiación del proyecto por parte de los participantes fue toda una sorpresa. Considerando que se trabajaba con voluntariado (JBC, 2016d).

La etapa final generó el acto de clausura (Figura 1), a partir de una experiencia de interpretación ambiental, creado con las PPR y el área de educación del JBC, donde se certificó la labor social realizada y se organizó un encuentro entre actores, en el que participaron víctimas del conflicto armado (CA), funcionarios del Estado y la sociedad civil, los cuales se escucharon por medio de propuestas artísticas que mostraron "una explosión de aptitudes y actitudes culturales, hubo poesía, fotografía, canto, exposiciones donde ellos explicaban lo que habían hecho" (E02). Así culminó el proceso, con un acto de entrega de un bosque de una hectárea a las víctimas, símbolo que dejó para la posteridad, el mensaje de que a pesar de todo lo vivido es posible hacer algo bueno, que crezca y se desarrolle de manera pacífica sin el obstáculo de la guerra. 
Fuente: Fotografías Autorizadas por el JBC

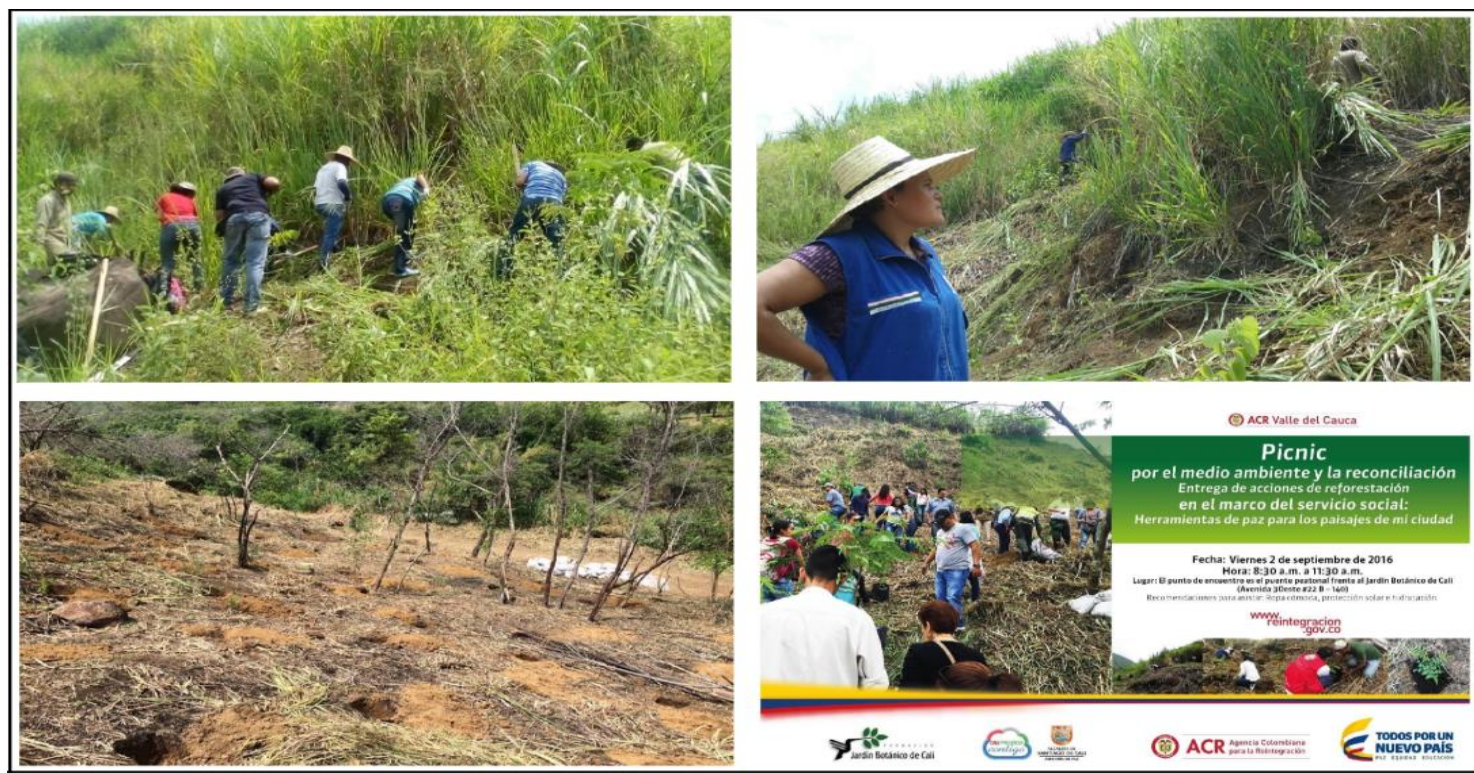

Figura 1. PPR en JBC

\section{Proceso 2017}

En este año la evolución del proceso cambió de forma, la ACR, a causa de la firma del Acuerdo de Paz, pasó a llamarse ARN, además entró la SPC, el Departamento Administrativo de Gestión del Medio Ambiente y la Secretaría de Deporte. En este periodo se desligó la Directora General, quedando a cargo del JB el Director Científico, pero el cambio más significativo se dio respecto a su razón social, pasando de JBC a Fundación Zoológica de Cali, aunque su operatividad mantiene cierta autonomía. En este proceso, el concepto de JB como territorio de paz (JB-UTP, 2016), consiguió consolidarse (E01, E02, E03), al considerarse "una herramienta para construir procesos de paz para la ciudadanía y para aquellos miembros que están en un proceso de reinserción” (E03).

El proceso se fortaleció en varios aspectos: 1) Se trabajó con PPR que ya habían cumplido 80 horas de labor social, concediéndoseles un incentivo económico por horas de trabajo; 2) Se continuó con la metodología HMP, incorporando además la metodología de Herramientas de Cultura Ciudadana (HCC); 3) La vinculación de instituciones del gobierno, permitió emplear 20 Gestores Culturales focalizando de una manera más directa la temática de Cultura Ciudadana. Nuevamente el proceso duraría 4 meses, incorporándose: la Subsecretaría de DDHH y Construcción de Paz, Gestores de Cultura Ciudadana para la Paz, Hojas Verdes y el DAGMA. 
En este proceso, se dio un crecimiento de la participación de la mujer, con 9 integrantes del total de 26 PPR. Bajo estos datos, se pudo tomar información de 3 mujeres (PPR: 1, 2, 3) (Tabla 1) y 5 hombres (PPR: 4, 5, 6, 7, 8). También se encontró una gran variedad de edades, que al compararlas con el tiempo de ruta de reintegración (Ruta), mostraba que la mayoría al salir de los GAOML no superaban los 22 años de edad. Por lo que se puede deducir que posiblemente han pertenecido a estos grupos por debajo de la minoría de edad, establecida en 18 años en el artículo 1 Convención sobre los Derechos del Niño (UNICEF, 2006) y el articulo 2 Protocolo Facultativo De La Convención Sobre Los Derechos Del Niño (Naciones Unidas, 2002), además los Convenios de Ginebra de 1949, protegen a todo niño menor de 15 años bajo medidas, regímenes preferentes y especiales ante la guerra en sus artículos: 14, 24, 38.5 y 76 (UNICEF, 2006). El reglamento de la Policía Nacional de Colombia, adoptado en enero del año 2000, prohibió la incorporación de niños. Los funcionarios civiles u oficiales militares que incumplan la prohibición de reclutar a menores de 18 años son culpables de mala conducta y pueden ser suspendidos (Human Rights Watch, 2003). Además, el Código Penal Colombiano, establece que, durante un CA, quien reclute menores de 18 años, incurrirá en prisión de 96 a 180 meses y multa de 800 a 1500 salarios mínimos legales mensuales vigentes (Art. 162. Ley 599 de 2000).

También podemos decir que la mayoría no terminó el bachillerato (PPR: 2-3-4-6-7), solo uno alcanzó a finalizar estudios de primaria (PPR7) y otro llegó al segundo grado de primaria (PPR6) (Tabla 1), evidenciando un bajo nivel educativo. Otro aspecto importante que se identificó, fueron las funciones laborales que realizaron y el lugar de trabajo (Art. 162. Ley 599 de 2000). De nuevo se pudo decir que la mayoría de PPRs son del Pacífico.

Tabla 1. Procesos Labor Social 2017.

\begin{tabular}{|c|c|c|c|c|c|c|c|c|c|}
\hline \multicolumn{10}{|c|}{ PROCESOS DE LABOR SOCIAL DEL JBC CON PPR DE GAOML 2017} \\
\hline GENERO & PPR & EDAD & $\begin{array}{c}\text { REGION } \\
\text { PROCEDENCIA }\end{array}$ & $\begin{array}{c}\text { TIEMPO RUTA } \\
\text { REINTEGRACIÓN } \\
\text { ACR/ARN }\end{array}$ & $\begin{array}{c}\text { NIVEL } \\
\text { EDUCATIVO }\end{array}$ & $\begin{array}{c}\text { FUNCIONES } \\
\text { QUE } \\
\text { REALIZAN }\end{array}$ & $\begin{array}{c}\text { LUGAR } \\
\text { DE } \\
\text { TRABAJO }\end{array}$ & HORARIO & $\begin{array}{c}\text { HORAS } \\
\text { LABOR } \\
\text { SOCIAL } \\
\quad \text { R }\end{array}$ \\
\hline \multirow{3}{*}{$9 \mathrm{M}$} & PPR1 & 19 & Tambo Cauca & 9 meses & Bachiller & $\begin{array}{c}\text { Limpiar, } \\
\text { marcar } \\
\text { árboles, } \\
\text { huecos, llenar } \\
\text { bolsas de } \\
\text { tierra rastrillar }\end{array}$ & $\begin{array}{c}\text { Ecoparque } \\
\text { de la vida, } \\
\text { Vivero y } \\
\text { Casa de } \\
\text { atención al } \\
\text { cliente JBC } \\
\end{array}$ & $\begin{array}{l}\text { Dom } 8 \text { am - } \\
\quad 4 \mathrm{pm}\end{array}$ & $80 \mathrm{H}$ \\
\hline & PPR2 & 21 & Tambo Cauca & 1,8 años & 9 Bachiller & $\begin{array}{c}\text { Limpiar } \\
\text { zonas, huecos, } \\
\text { limpiar ribera } \\
\text { del río }\end{array}$ & $\begin{array}{l}\text { Rio Cali y } \\
\text { Ecoparque } \\
\text { de la vida }\end{array}$ & $\begin{array}{l}\text { Dom } 8 \text { am - } \\
\quad 4 \mathrm{pm}\end{array}$ & $80 \mathrm{H}$ \\
\hline & PPR3 & 23 & Cali & 3,5 años & 10 Bachiller & $\begin{array}{l}\text { Limpiar y } \\
\text { recoger } \\
\text { basura, } \\
\text { limpiar } \\
\text { canales, }\end{array}$ & $\begin{array}{c}\text { Ecoparque } \\
\text { de la vida, } \\
\text { Rio Cali, } \\
\text { Vivero, } \\
\text { Casa de } \\
\end{array}$ & $\begin{array}{l}\text { Lun y Dom } \\
8 \text { am - } 4 \text { pm }\end{array}$ & $80 \mathrm{H}$ \\
\hline
\end{tabular}




\begin{tabular}{|c|c|c|c|c|c|c|c|c|c|}
\hline \multicolumn{10}{|c|}{ PROCESOS DE LABOR SOCIAL DEL JBC CON PPR DE GAOML 2017} \\
\hline \multirow[t]{2}{*}{ GENERO } & PPR & EDAD & $\begin{array}{c}\text { REGION } \\
\text { PROCEDENCIA }\end{array}$ & $\begin{array}{c}\text { TIEMPO RUTA } \\
\text { REINTEGRACIÓN } \\
\text { ACR/ARN }\end{array}$ & $\begin{array}{c}\text { NIVEL } \\
\text { EDUCATIVO }\end{array}$ & $\begin{array}{c}\text { FUNCIONES } \\
\text { QUE } \\
\text { REALIZAN }\end{array}$ & $\begin{array}{c}\text { LUGAR } \\
\text { DE } \\
\text { TRABAJO }\end{array}$ & HORARIO & $\begin{array}{c}\text { HORAS } \\
\text { LABOR } \\
\text { SOCIAL } \\
\text { R }\end{array}$ \\
\hline & & & & & & $\begin{array}{l}\text { empacar } \\
\text { bolsas de } \\
\text { tierra, recoger } \\
\text { residuos de } \\
\text { guadaña }\end{array}$ & $\begin{array}{l}\text { atención } \\
\text { visitante }\end{array}$ & & \\
\hline \multirow{5}{*}{$17 \mathrm{H}$} & PPR4 & 41 & Buenaventura & 4 años & 7 Bachiller & Hacer huecos & $\begin{array}{l}\text { Ecoparque } \\
\text { de la vida, } \\
\text { Terminal } \\
\text { de buses }\end{array}$ & $\begin{array}{l}\text { Dom } 8 \text { am - } \\
\quad 4 \mathrm{pm}\end{array}$ & $80 \mathrm{H}$ \\
\hline & PPR5 & 30 & Orito Putumayo & 2,3 años & Bachiller & $\begin{array}{l}\text { Huecos, } \\
\text { siembra, } \\
\text { abonar y } \\
\text { plantear }\end{array}$ & $\begin{array}{l}\text { Ecoparque } \\
\text { de la vida }\end{array}$ & $\begin{array}{l}\text { Dom } 8 \text { am - } \\
\quad 4 \mathrm{pm}\end{array}$ & $80 \mathrm{H}$ \\
\hline & PPR6 & 25 & Curillo, Caquetá & 3,4 años & 2do Primaria & $\begin{array}{l}\text { Huecos, } \\
\text { siembra, } \\
\text { abonar y } \\
\text { plantear }\end{array}$ & $\begin{array}{l}\text { Ecoparque } \\
\text { de la vida }\end{array}$ & $\begin{array}{l}\text { Dom } 8 \text { am - } \\
\quad 4 \mathrm{pm}\end{array}$ & $80 \mathrm{H}$ \\
\hline & PPR7 & 26 & Argelia Cauca & 4,7 años & primaria & $\begin{array}{l}\text { Huecos, } \\
\text { siembra, } \\
\text { abonar y } \\
\text { plantear }\end{array}$ & $\begin{array}{l}\text { Ecoparque } \\
\text { de la vida }\end{array}$ & $\begin{array}{l}\text { Dom } 8 \text { am - } \\
\quad 4 \mathrm{pm}\end{array}$ & $80 \mathrm{H}$ \\
\hline & PPR8 & 24 & Guapi & 2,1 años & Bachiller & $\begin{array}{c}\text { Limpiar, hacer } \\
\text { huecos, } \\
\text { platear, } \\
\text { construir } \\
\text { semillero }\end{array}$ & $\begin{array}{c}\text { Ecoparque } \\
\text { de la vida, } \\
\text { Maloca } \\
\text { JBC, Rio } \\
\text { Cali }\end{array}$ & $\begin{array}{l}\text { Lun, Mar, } \\
\text { Sáb y Dom } \\
8 \text { am - } 4 \text { pm }\end{array}$ & $80 \mathrm{H}$ \\
\hline
\end{tabular}

Fuente: Elaboración propia.

Al final del proceso, "se realizó un evento en el cual se entregó diplomas, certificados, un evento más en sentido de gratitud hacia la labor que ellos han desempeñado y donde básicamente se busca que la comunidad conozca qué se ha hecho con estas personas" (E03). Lo bueno que logró el JBC, fue seguir "brindando espacios y posibilidades para que las personas que quisieran hacer servicio de labor social en el JBC, pudieran seguir apoyando las actividades misionales” (E03).

\section{Proceso 2018}

Bajo la gestión de la Fundación Zoológica, se lograron diez jornadas (Tabla 2) de servicio social: dos canceladas, dos situaciones críticas de manejo especial y seis que se desarrollaron sin novedades. "De las jornadas que se han podido realizar, tres han sido en el área de restauración ecológica del Predio Piedragrande, tres en la Loma del Bosque Municipal y dos en comunidades del sector” (E01, JBC 2018). 
Tabla 2. Actividades JBC 2018

\begin{tabular}{|c|c|c|c|}
\hline \multicolumn{5}{|c|}{ ACTIVIDADES JBC 2018 } \\
\hline FECHA & ÁREA DE TRABAJO & ACTIVIDADES & N$^{\circ}$ PPR \\
\hline $18 / 02 / 2018$ & Predio Piedragrande & Siembra de Árboles & 22 \\
\hline $25 / 02 / 2018$ & Loma del Bosque Municipal & $\begin{array}{c}\text { Reconocimiento del terreno, deshierbe } \\
\text { selectivo, plateo con cartón a nacederos. }\end{array}$ & 18 \\
\hline 4/03/2018 & Predio Piedragrande & $\begin{array}{c}\text { Deshierbe selectivo, plateo de árboles, control } \\
\text { de pasto, búsqueda semillas carbonero en canal } \\
2 .\end{array}$ & 19 \\
\hline $11 / 03 / 2018$ & Predio Piedragrande & Plateo de árboles, deshierbe selectivo. & 18 \\
\hline $25 / 03 / 2018$ & Barrio La Fortuna & Plateo de árboles, deshierbe selectivo. & 18 \\
\hline $8 / 04 / 2018$ & Quebrada Pilas del Cabuyal & $\begin{array}{c}\text { Apoyo minga comunitaria de limpieza de la } \\
\text { quebrada Pilas del Cabuyal. }\end{array}$ & 12 \\
\hline $15 / 04 / 2018$ & Ninguna & La actividad se canceló. & 0 \\
\hline $22 / 04 / 2018$ & Loma del Bosque Municipal & $\begin{array}{c}\text { Limpieza de material vegetal, apertura de } \\
\text { huecos. }\end{array}$ & 12 \\
\hline $29 / 04 / 2018$ & Loma del Bosque Municipal & Guadañada de zonas y apertura de huecos. & 12 \\
\hline $6 / 05 / 2018$ & Ninguna & Se cancela actividad. & 0 \\
\hline
\end{tabular}

Fuente: Elaboración Propia.

\section{Punto de vista de desmovilizados: Narraciones de PPR}

Un aspecto que se pudo indagar es la ruta realizada de las PPR antes de llegar al JBC (Figura 2) como se demostrará a continuación.

Fuente: Elaboración propia a través de Google My Maps.

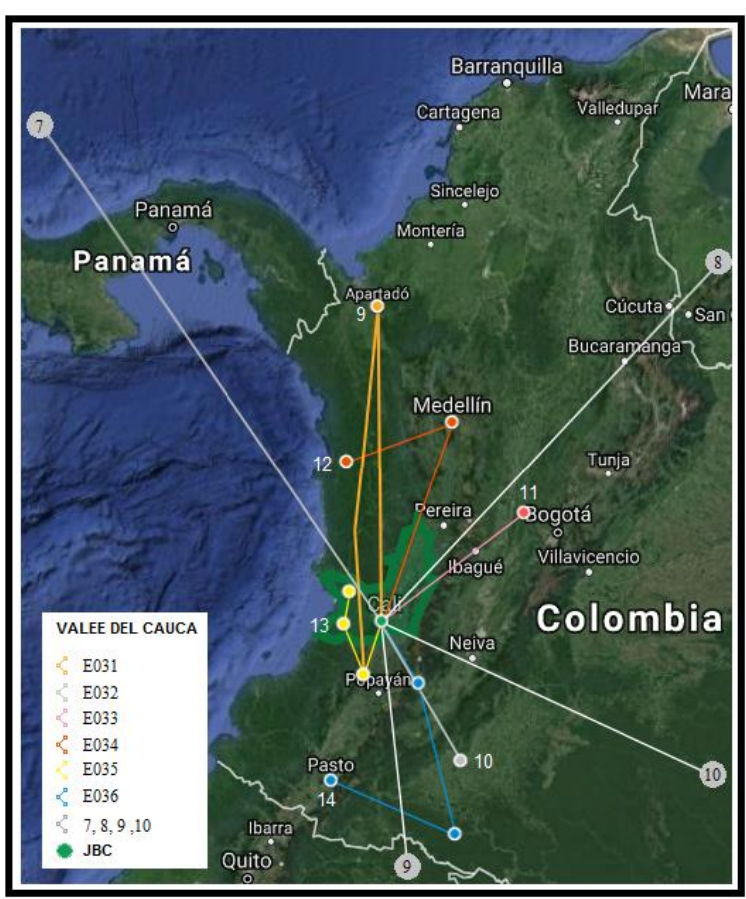

Figura 2. Movilidad 


\section{Víctima}

La víctima (E04), línea naranja (Figura 2), se movilizó por un suceso que pasó con un hermano, "por medio de la guerrilla, le llegaron varias amenazas, por lo que él no quiso cumplir con unas peticiones y fue cuando amenazaron a toda la familia" (E04), la familia migró hacia la Costa Atlántica y él PPR hacia Apartadó (Urabá).

Estando en Urabá, nuevamente es víctima del CA. El evento se produjo al estar trabajando en una empresa comercializadora de banano que no cumplía con el "impuesto revolucionario" que imponía el respectivo grupo guerrillero en la zona y "amenazaron a los empleados y me tocó desplazarme" (E04). Esto lo llevó a una nueva decisión ante la inestabilidad laboral y ambiente criminológico a buscar nuevas alternativas en "Miranda, Apartadó, Tumaco, Huila y por último Cali" (E04), demostrándose de esta manera, que una víctima al ser desplazada de su lugar de origen, trabajo o familia, ante su vulnerabilidad, se ve en la necesidad de asumir acciones de movilidad nómada, ante la falta de cubrimiento de necesidades básicas y de seguridad.

Una de las consecuencias "fue la desintegración de la familia, que es algo que uno no supera fácilmente porque pierde lo espiritual, moral, familiar, material, la tranquilidad y la parte psicológica" (E04), pero aun así, tras el proceso en el JBC, la víctima pudo superar esa faceta, "al inicio fue un poco duro llegar a ese acercamiento después de lo que había pasado y tener que compartir, sabiendo que de pronto una de estas personas participó en los agravios que vivió nuestra familia" (E04), pero en estos procesos la mentalidad cambia a medida que se van conociendo unos a otros por medio del trabajo y las horas voluntarias. "Gracias a Dios que me dio la fortaleza para vivir esta situación con ellos y poco a poco me iba haciendo ideas de aceptación y de perdón" (E04). El trabajo, el tiempo y la cercanía permite "hacerles muchas preguntas y al final uno se da cuenta que son cosas que suceden y que hay que darle un manejo a esa situación, entonces al final entablamos una amistad con muchos de ellos, entonces ya el concepto fue otro" (E04), siendo esta una clase de reconciliación directa de parte de personas desmovilizadas a una víctima y un cambio de mirada y de aceptación de parte de la víctima a quienes han sido victimarios.

\section{Exguerrillero}

El guerrillero (E05), línea gris (Figura 2), ingresó a las filas de la guerrilla sin haber cumplido la mayoría de edad. Para él, ser guerrillero es "mirar las necesidades de un pueblo, es luchar por un pueblo para una igualdad" (E05). Como exguerrillero, es consciente que pertenecer a 
un grupo genera experiencias, "en lo positivo aprendí a ser muy organizado, el grupo es muy estricto. Lo negativo es causar daño a otras personas que no le han hecho daño a uno, en ataques, en tomas" (E05). Algo bastante negativo para un menor, es utilizar armas de fuego, “inclusive uno desde que entra allá lo van induciendo a cómo debe manejarla y cómo tiene uno que hacer" (E05).

Y aunque se pueda hablar positivamente de las leyes, normas, organización y estatutos, "la justicia es muy drástica, no se permiten ladrones, violadores, atracadores, ni tampoco dentro de los grupos, al que llegue a cometer dicho error se le sanciona con el fusilamiento por consejo de guerra" (E05). Así pues, existe un discurso del miedo, quien cometa un error puede morir.

Un menor, ante estos acontecimientos ¿qué puede sentir o pensar? ¿qué impulsos desarrollará a medida que su pensamiento va madurando? lo cierto es que este menor en muchas ocasiones se sentía extraño de sí, "pienso que me sentía como ya siendo un adulto mayor siendo aún un adolescente" (E05), en medio de esos círculos, los menores pierden la infancia y el derecho a ser niños, "uno estando en ese lugar ya no va a decir que en su niñez compartió con sus amigos, con niños de su misma edad, jugó, se divirtió en el colegio, le deja secuelas a uno" (E05).

En estos casos, es muy difícil que un menor deserte, el menor se va adaptando y aceptando su realidad, por ello, lamentablemente, una de las formas para que vuelvan a la vida social es cuando son heridos o atrapados. Este PPR lo recuperaron en Caquetá, "salí de los lados de La Montañita, me trasladaron para Florencia a un hogar tutor y de allí para Cali a seguir con la ruta de restablecimiento de derechos con la ARN" (E05), es decir, este menor fue un desvinculado (ARN, 2019), no pasó por los procesos de los demás entrevistados sino que fue tratado como menor, no como victimario sino como víctima del CA. Esto permitió que, al llegar a su mayoría de edad, pudiera emprender su Ruta, en donde le dieron vía para trabajar “en jardinería, en una empresa de Cali” (E05). De allí, pasó al JBC en donde fue seleccionado por la ACR para "cumplir el deber de aportar a la sociedad 80 horas de trabajo" (E05). En el JBC, le tocó trabajar con "siembra de árboles, limpiar plantas, empacamiento de bolsas y abono" (E05) y en la zona de las oficinas, "replantamos árboles para limpiar el sector, guadañarlo y respectivamente volvimos y los colocamos en su lugar, en diez días terminé mis ochenta horas, cada domingo de ocho horas" (E05). 
Nuevamente, volvió a sentirse alegre, a compartir con otras personas y socializar, "me sentí bien, agradable, lo de plantas me gusta" (E05), todo esto lo que demuestra, es que estos procesos, sin importar edades, hacen que una persona pueda volver a rehacer su vida de una forma positiva, "pienso que, como estoy empezando otra vez mi vida es totalmente bien, me siento bien con lo que hago" (E05). Actualmente tiene una pareja, se le nota alegre, con buen semblante y dada la experiencia que ha obtenido en jardinería y el prestigio del JBC, ya le han ido saliendo nuevos trabajos y ha empezado a tomar decisiones positivas para su vida, "para mí ha sido un cambio completamente desde el momento en que me recuperaron de allá. He empezado a trabajar por mi vida dejando atrás el pasado que me ha marcado, entonces he comenzado a preocuparme por hacer las cosas bien" (E05). Volver a la vida civil para este joven, es algo que han mencionado la mayoría de las personas entrevistadas "es como volver a nacer" (E05).

\section{Exguerrillera}

Esta guerrillera (E06), línea rosa (Figura 2), es una de tantas mujeres que han pertenecido a la guerrilla en el Valle, una menor más, que por circunstancias de abusos y pobreza le tocó salir de su hogar huyendo de la mala situación que tuvo que soportar, "en el tiempo en que yo estuve entraron muchas menores de edad, le lavan la cabeza, cuando yo me fui nos fuimos catorce y todos éramos menores" (E06), esto es debido a la gran inequidad que sufre Colombia:

"Eso también depende del sitio en donde uno se crie y en donde uno esté, de pronto en el lugar en donde yo estaba había mucha gente humilde, donde los padres no son muy pendientes de sus hijos, había mucha guerrilla, hacían reuniones al aire libre y todo el mundo podía ir" (E06).

Es así como salen de situaciones difíciles para adentrarse en un mundo donde los derechos humanos son vulnerados, un mundo jerárquico y disciplinario, ordenado, "eso viene por etapas, cuando uno entra pues uno es raso y a medida que pasa el tiempo uno va obteniendo cargos, fui comandante de escuadra" (E06), en cierto sentido suena muy bien, genera una especie de empoderamiento, pero sin salario, prestaciones y vacaciones, sin días festivos, "es trabajo duro, pero uno asimila lo que toca (...) pero de pronto que sean mejores personas no creo, porque igualmente uno tiene que tener al hombro un fusil y es a las órdenes que le den a uno" (E06). Allí es donde se sumergen en la guerra, "me ha tocado combatir en lo rural contra las fuerzas armadas y el paramilitarismo, mi primer fusil un AK47 y luego una M16, en su momento uno se siente como Rambo" (E06), indudablemente si existe un cambio real y un 
empoderamiento del ser, pero a qué costo, porque por mucho que un GAOML intente resolver el CA, con ello genera conflictos subjetivos.

Uno de los casos más duros que tiene que vivir la mujer en las filas de la guerrilla, es tener un hijo, porque es casi prohibido quedar en embarazo, se viola el derecho a la vida, siendo esto un punto de inflexión para tomar una decisión de abandono de un GAOML "es duro, porque uno portando un fusil y el equipo al hombro y uno en embarazo, pero a seis meses de que estaba en embarazo salí a la civil a tener mi bebé" (E06) y de aquí vino el escape de las filas, a sabiendas lo que puede generar un consejo de guerra, "sí, ya es para pelona, pues como se dice, que los matan" (E06). Otro derecho violado es el de la información, no se permitían escuchar las noticias y menos de desmovilización, "nos decían que eso era una mentira, que después nos iban a meter a la cárcel, pero eso no fue así, yo me entregue al GOES” (E06), los Grupos Operativos Especiales de Seguridad. El proceso pasa por ciertos tiempos que se tienen que cumplir, "primero lo llevan a un médico, toman fotos para saber si uno tiene golpes, preguntan nombres, de que frente, exactamente todo y de allí a uno lo mandan a un albergue antes de entregarlo al CODA" (E06), el Comité Operativo para la Dejación de las Armas (ARN, 2019, p. 8), genera un certificado que hace constar que una persona se ha desmovilizado.

Un primer paso es aislar al PPR de la región donde se entrega:

"Estuve en Bucaramanga en un albergue como máximo tres o cuatro meses esperando el CODA, estuve con mi hijo, fue una experiencia muy chévere, del CODA me trasladaron a Cali, le dan una bonificación para que uno esté los primeros meses bien donde uno vaya a estar y de allí empieza el proceso de reintegración paso a paso" (E06).

Aquí inicia el proceso con la ACR después de comprobada toda la información pertinente y veraz acerca del desmovilizado:

"Ya le dicen a uno los beneficios que uno tiene, uno firma un acta confirmando que después de que uno firme eso no lo pueden ver a uno delinquiendo y tiene que estar bien en su proceso hasta la terminación y el proceso empieza por los estudios y la culminación es tener ochenta horas de labor social" (E06).

La agencia se encarga de buscar alguna institución que les brinde la posibilidad de realizar este requisito "y pues gracias a Dios el JB nos dio la oportunidad de venir hacer labor social" (E06). Ahora esta exguerrillera está rehaciendo su vida después de haber culminado su Ruta, "ahora sigo con lo de la peluquería, pero estoy buscando trabajo" (E06). 


\section{Excomandante miliciano afrodescendiente}

Este miliciano (E07), línea roja (Figura 2), ingresó en la guerrilla por medio de un amigo. En la guerrilla se hizo comandante de milicia "tenía yo mi casa, yo de allí salía, me tocaba irme al monte cuando venía el ejército, una vez, se metieron los paramilitares y me la iban a quemar" (E07). Esto evidencia una forma estratégica de operar:

"Como yo hice muchas cosas buenas en la guerrilla, yo ayudé mucha gente, entonces estaban pilas y apenas venía el ejército me llamaba (...) yo no salía de allí y cuando me desmovilicé de mi casa salí (...) yo solo iba a los campamentos cuando me llamaban a reunión" (E07).

La realidad de un comandante de milicia es pertenecer a un territorio, hacerse responsable de determinados encargos y sobre todo vigilar y controlar una región, por tanto, pierde el derecho a la libertad, pero a su vez, esta privación, genera un proceso decisorio:

"Yo no podía salir, me perseguía mucho la ley, no podía salir al pueblo, a la sociedad, para ningún lado, porque enseguida me buscaban, entonces me desmovilicé, llegué a Quibdó el 13 de mayo de 2013 a un batallón, estuve mes y medio y de allí me mandaron para Medellín al Hogar de Paz a estudiar y comenzar la primaria” (E07).

Este proceso de desmovilización, en las filas de la guerrilla, se llama desertar, determina escapar de un GAOML, poniendo en estado de vulnerabilidad a toda persona que lo realiza, convirtiéndose en objetivo militar.

Después de vivir en Medellín, el PPR solicitó traslado a Cali, por medio de la ACR, "entonces un día estando en la casa me llamaron, dizque que me necesitaban en el JB para trabajar una semana, me presenté y ese mismo día comenzamos, llegamos como dieciocho y solo quedamos dos" (E07), el trabajo de desyerbar el pasto elefante fue una labor tan ardua, que nadie se quiso quedar, "le sacamos la paja a los árboles, nos mandaron a caciquear, sembrar, abonar" (E07), siendo esa semana "la más dura y la mejor, porque por eso estoy aquí" (E07). Dicho trabajo, después de haber estado dos años en Medellín, terminar primaria y recibir una ayuda del gobierno de 160.000 pesos mensuales, le permitió finalizar su Ruta. Ante ello el miliciano manifestó que la decisión de volver a la vida civil ha sido:

"Lo mejor, porque uno en un grupo lleva todas las de perder, lo pueden matar, llevar a la cárcel, mirar con desprecio, a mí me tocaba darme cuenta que iban a matar a alguien 
o me mandaban a matar gente, gente que vivió conmigo alrededor y yo le daba el pasaje para que se fuera" (E07).

Pero como volver a la vida civil, es volver a la legalidad, "porque la guerrilla secuestra, extorsiona, pide vacuna" (E07), la decisión de cambio tiene mucho que ver con desistir al delito y optar una acción pro social.

\section{Excomandante guerrillero}

Este excomandante (E08), línea amarilla (Figura 2), centra su historia en el Pacífico, "cuando salí de mi casa, yo vivía en la parte rural de Buenaventura, cuando yo llegué al grupo, el grupo continuó allí unos 8 meses, luego para los Farallones, allí tuvimos un año largo y ya nos desplazamos hacia el pacífico porque ya la zona se calentó" (E08), toda esa zona es montañosa y de difícil acceso:

"Bajamos por el Alto de Anchicayá, por la vaina de los diputados, a mí no me tocó, pero estaba en la zona, eso le tocó a la gente que llevaba más tiempo y conocían la ciudad. Ya en el pacífico nos desplazábamos por diferentes ríos: Mayorqui, Cajambre, Yurumangui, Naya, Micay y luego parte del Cauca” (E08).

Al decidir desmovilizarse se vincula a la ACR de Cali y por medio de esta agencia entra al JBC, "con la hoja de vida y como había trabajado dos semanas en el JB, la jefa tuvo eso en cuenta para llamarme" (E08), estando en el JBC el desconocimiento de las personas era total, "yo vine solo, pero entonces hablando con los compañeros me di cuenta que eran de grupos armados, el integrador sabía que venían otros compañeros del programa y que eran guerrilleros que no los conocía” (E08).

Esto llevó al guerrillero a cambiar totalmente su vida y a pensar de otra manera, "es un paso importante, me sentí bien, satisfecho de estar en esta vida, digamos cambiar ese modo de vida que tenía" (E08), así pues, se pudo constatar, que durante su estancia en la guerrilla se le violó su derecho a la educación, "pude terminar los estudios y ahora en abril terminé mi bachillerato, estudié en un acelerado" (E08), además, también se le violó sus derechos laborales al tener que ejercer labores y responsabilidades sin tener alguna compensación salarial.

Un aporte importante que se pudo analizar fue el concepto de Campesino Amarrado:

"Es cuando se coge a la persona, se amarra y se ajusticia, se lo llevan para la montaña, hacen el hueco, lo entierran y lo dejan allí, hay demasiados cadáveres en las montañas, 
porque hay mucha gente que se ajustició, digamos miles de cadáveres, porque en toda parte donde los grupos llegan suceden esas cosas" (E08).

Todo esto va en relación a la forma de aplicar la justicia en las filas guerrilleras por medio de Consejos de Guerra:

"Se hace una asamblea donde se reúne la gente y entonces lo tiran a voto, para llegar a ese extremo es porque hay un delito como robo, asesinato, "el guerrillero cometió tal delito, quienes están de acuerdo para que sea fusilado", entonces si la mayoría levanta la mano se hace el hueco, lo matan y lo entierran" (E08).

\section{Exguerrillero Indígena}

Este indígena (E09), línea azul (Figura 2), muestra una visión esclarecedora de los procesos de desmovilización:

"Se podría decir que uno en dos años ya estaba en libertad, ahora el proceso cambió, uno tiene que durar dos años en seguimiento y de allí hay muchas formas, anteriormente con quinientas o seiscientas horas ya recibía un proyecto productivo, hoy en día no, una persona si es analfabeta tiene que terminar sus estudios para que tenga beneficio de proyecto" (E09).

Estos procesos eran los que se daban entre el 2000 y el 2004, “el mismo Estado lo obligaba a uno, toma este proyecto o pierde todo, entonces uno tenía que decir, venga pa' acá" (E09).

Este PPR a la edad de cinco años fue regalado y enviado a Cali, Bogotá y por último a Nariño, en Nariño "cogí sentido, tendría mis trece años, y como hay un dicho, las paredes tienen oídos, pues yo esta po' allí, cuando una señora dice "pero este muchachito se amañó a pesar de que lo cogimos pequeñito y mire que se adaptó” (E09). Para ese entonces vivía con la persona que lo crió y le brindó "calor humano, me consentía, ya tenía también sus años y de un derrame cerebral murió" (E09).

Al alcanzar los dieciséis años, vendió un ternero en 11.000 pesos y se dirigió al Putumayo:

"Me fui sin conocer nada, venían de Sucumbí o de Churuyaco, eran seis o siete que cuidaban la zona e hicieron una reunioncita en Siberia, antes de llegar al río Guames. Empezaron a hablar que como el rico explota al pobre, que como engaña y quita sus hijos, que se los llevan, dicen que les dan estudio y lo que hace es hacer trabajar y por eso están así, y bueno, todo eso era como que me caía a mí y dije, yo soy uno de ellos, 
entonces po allí se descuidó uno y le dije "bueno, que requisitos uno necesita" y me dijo "nada, es la voluntad, usted quiere seguir con nosotros", le dije "si, porque lo que dicen es verdad" (E09).

En ese momento entra a las filas del M-19, moviéndose por el Putumayo:

“San Miguel, La Dorada, La Hormiga, Santa Ana y de allí me vine pa' el Cauca, pero yo siempre dentro de las filas pensaba, yo tengo que encontrar a mi familia, entonces allí fue donde hice la búsqueda y entré pa' el Cauca, cuando se hizo la toma de Inzá, La Plata, se hicieron muchas cosas, allí es donde yo encontré a mi familia" (E09).

Pero el reencuentro no fue muy bueno, no lo reconocieron, “y encontrándome con la familia me sentí mal" (E09), además, en las comunidades indígenas son estrictos respecto al CA, y mucho más después de la desmovilización del Movimiento Quintín Lame, “yo pertenezco a una etnia indígena Páez, onde hay un reglamento onde dice que las personas que se aigan ido a grupos armados son personas que son desconocidos" (E09). Esto lo que genera, tras una decisión de abandono de GAOML es una triple victimización al desmovilizarse a pesar de ser victimario: 1) El ser excombatiente lo aísla del común por estigmatización social; 2) No se puede llegar donde la familia porque es perseguido como desertor y 3) Si se ha demostrado que ha sido guerrillero, no puede volver nunca a ser parte de su comunidad étnica, tan solo de visita si demuestra ser desmovilizado.

En el año 1992, intentó desmovilizarse con el M-19, pero el proceso con el Estado no se cumplió y lo que pudo ser una decisión de abandono se convirtió es una decisión de reincidencia:

“Tenía unos 27 años y para mí eso era como cosa de no rendirse, entonces el mismo comandante decía, "los que quieran quedarse todavía al frente del combate, salir de las filas de formación", entonces salimos quince y quedó un responsable de la zona de Tierradentro, quedó encargado de las mejores armas que habían, todas las viejas fueron entregadas al consejero de paz" (E09.).

Es muy conocido que, para ese tiempo, al entregarse las armas se inició una cacería de brujas, el gobierno prometió llevar la paz al campo, pero nunca llegó y llegaron nuevas fuerzas y delincuentes, "entonces allí ya tocaba, como uno ya tenía la dotación y la experiencia, defender esos territorios" (E09). 
Al final, él entrevistado manifiesta que termina en las FARC, donde le tocó desmovilizarse. "Yo no deserté me cogieron entre Toribio y Piendamó, en un punto que llaman Alto Grande, allí me capturaron, a uno le meten psicología, "usted como tiene orden de captura en el 81 y cayó en 2003, usted mejor dicho cadena perpetua" (E09). Después de su captura, terminó todo su proceso en el 2006 con otros desmovilizados:

“Allí nuevamente alguna juventud no acató y empezaron a integrarse a otros grupos, entonces el gobierno nuevamente nos pasó un comunicado que tenían que recoger a todos y nos metieron al proceso, allí ya la cosa es seria, porque nos investigaron, ya donde uno iba tenía que tener un soporte, ¿qué estaba haciendo? ¿dónde estuve? pero yo no he tenido inconveniente, desde que salí de allá he sido una persona que ha trabajado con honestidad" (E09).

Así fue como llegó al JBC, demostrando que, por mucho tiempo que permanezca una persona en un GAOML, es posible decidir volver a la vida civil sin importar las circunstancias del abandono del GAOML, "para mí fue como volver a empezar de cero, porque en la vida civil uno cuando vuelve no tiene nada, ni un rancho donde vivir, si es una ciudad pues tiene que pagar un arriendo" (E09). Lo mejor de todo, es que después de tantas experiencias, hoy en día está con su mujer y sus hijos, gran punto de inflexión para desistir al delito. Para el 31 de diciembre de 2017, pudo pasar por primera vez una navidad con su familia, después de 52 años de estar alejado de sus padres.

\section{Labor social de PPR en el JBC}

Como ya es sabido, los procesos de reintegración no se realizan por medio del JBC, sino por medio de la ACR hoy en día ARN. Por ello, lo que realmente se hace en el JBC es servicio social voluntario, el cual, es necesario para las PPR que deben "hacer su reinserción final en el mundo laboral. Es un requisito indispensable que debe cumplir una persona que se está reintegrando a la sociedad antes de entrar de nuevo a la vida civil" (E03). Estos procesos, lo que han generado es que al finalizar el año 2016, se pudiera "contratar a dos de las PPR" (E01), es decir, dos de las 27 PPR que estuvieron haciendo su labor social, siendo la ACR quien "monitoreó el trabajo" (E02).

Un aspecto que se evidenció, es la ausencia de derechos laborales, "el mejor trabajo que he tenido es este, ya que en la guerrilla no le pagan a nadie, los únicos que pagan son los paramilitares, en la guerrilla los que usan plata son los comandos, el guerrillero raso solamente recibe comida y dotación” (E07). Así también lo manifiesta otro PPR, "yo nunca 
había trabajado con ninguna entidad, solo cuando llegué aquí empecé a conocer lo que se manejaba en el JB, me sentí muy bien" (E08).

Una de las acciones que más generan cambios cognitivos y transformación de identidad para asumir decisiones de parte de los desmovilizados y que pueden ejercer las instituciones, son las actividades de carácter pro social. Ante ello, el JBC fortaleció este aspecto vinculando a los desmovilizados en distintos lugares de trabajo durante los tres procesos que se generaron (Figura 3). Se puede decir que en el 2016 solo se trabajó en el JBC, para el 2017, "estuvimos manejando cinco jardines afuera, donde sembramos árboles, hicimos limpieza y manteamiento" (E08). Los lugares de trabajo fueron: 1) Ecoparque de la Vida; 2) Bosque Municipal; 3) Parque Público del Peñón; 4) Parque Público Terminal de Transportes y 5) Zonas Verdes Barrio la Fortuna, este último, fue importante en el 2018, dado que el proceso pasó de local a comunitario, siendo este contexto de vital importancia, pues es en la comunidad en donde se genera un reconocimiento del individuo y en donde éste se reconoce como actor social.

Fuente: Elaboración propia a través de Google My Maps

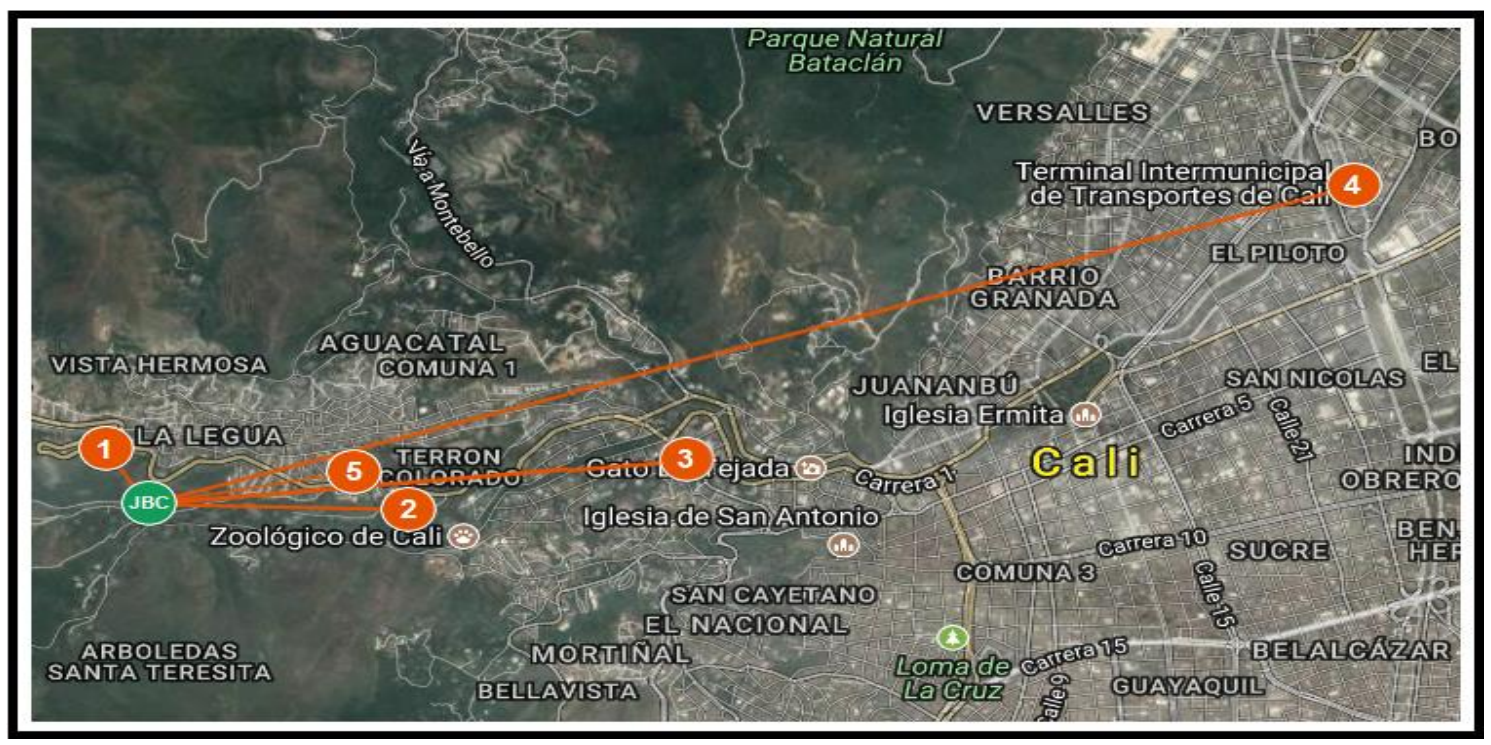

Figura 3. Movilidad Laboral en Cali de PPRs

Sin embargo, aún existen algunos tabúes de parte de las personas en general, en cuanto a las PPR en su forma de ser y de relacionarse, pero se pudo demostrar que estas personas son buenas para cualquier labor:

"Hay muchos actores de la sociedad que encuentran cierta reticencia y prevención, ya que desafortunadamente el conflicto generó muchos dolores, abrió brechas en la 
sociedad y hay sectores que sienten que ellos no deberían tener ningún perdón ni que se les debería dar alguna oportunidad de reinsertarse en un proceso de sociedad" (E03).

En el JBC, las percepciones negativas y desconfianzas han desaparecido, por el contrario, al venir de lugares totalmente conflictivos y brindar el JBC una oportunidad para que culminen su proceso, se logró fusionar conocimientos, "yo como venía de trabajar con cultivo comercial: banano, palma africana (...) se me da la oportunidad de venir a trabajar acá con plantas menores con otro tipo de manejo de agricultura" (E04), por tanto, se ha alcanzado a establecer vínculos entre el conocimiento institucional y comercial, transformándolos en herramienta "para que se logre entender que la vida va más allá de empuñar un arma y de luchar por ideales, la sociedad y la vida se construyen a partir de las relaciones entre seres humanos" (E03).

Como es normal, las PPR también llegan prevenidos "por el rechazo de varios sectores, a veces, es muy difícil entrar en una conversación, son muy introvertidos, no te sostienen una mirada, son personas que, de cierta manera, se sienten como menores" (E03) pero en el JBC se ha utilizado el escenario, circunstancias y condiciones para poder hacerlos sentir bien consigo mismos ya que son personas "que realmente pueden hacer mucho por la sociedad. Hay algunos que tienen mucha disposición para el trabajo, digamos que son personas que quieren sentirse útiles" (E03).

Todo esto ha permitido, que tanto víctimas como victimarios de varios grupos trabajen unidos:

“A mí me tocó coordinar más o menos unas 30 personas, cada integrante tenía que pagar ochenta horas, un grupo que no se imaginaba uno que eran personas que habían estado en ese mundo, porque la parte humana y laboral es diferente, muy ordenados, disciplinados, incluso estaban prestos y dispuestos a lo que surgiera, entonces hubo mucha dinámica y mucha participación” (E04).

Esto es lo que logran estos procesos, que víctimas, victimarios y personal institucional vean y presencien el otro lado del ser humano, son "personas maduras, estaban muy conectadas con lo que querían, se trabajó con mucho agrado, las personas se prestaron para ello" (E04), demostrando de esta manera que dentro de otro ambiente estas personas pueden realmente aportar a la sociedad, "han podido reincorporarse de una forma muy fácil a la vida civil, 
incluso se les ve la educación, la participación, la colaboración, se atreve uno a decir que es muy notorio el cambio" (E04)

Otro aspecto muy interesante ha sido trabajar con mujeres:

"Son realmente muy berracas, muy fuertes. Muchas de ellas han tenido unas historias difíciles, pero las veo con disposición de salir adelante, sentirse útiles, construir familia, reinsertarse en un proceso de sociedad, las veo muy dispuestas a trabajar, a superarse, a olvidar lo que pasó y ver un futuro más prometedor" (E03).

En ningún momento se determinó una división del trabajo por género, "todos por igual" (E06), tanto hombres como mujeres:

"El primer día, fuimos carretera arriba por todo el río recogiendo escombros y basura que deja la gente los fines de semana y de allí me tocó lo que fue limpiar canales, arriba en el filo punteando y en el vivero empacando tierra, aquí en la casita del JB recogiendo escombros" (E06).

De las mujeres que cumplieron el tiempo de las ochenta horas de labor social, "las labores eran por igual, "yo voy a bolear machete", "yo voy a tirar pica", "yo voy a cortar matas", "yo voy a cargar la guinea" y eso se hacía” (E04), en sí, aunque es normal que exista una división del trabajo entre hombres y mujeres, por lo general en trabajos de campo, las mujeres siempre demostraron que si tenían que hacer una labor dura lo hacían.

\section{Conclusiones}

I. Se logró analizar una evolución significativa, evidenciando que, durante el proceso de 2016, se inició un trabajo participativo y colaborativo entre el JBC y la ACR de modo institucional. Para el 2017, se alcanzó una dimensión local objetiva, al trabajar las PPR tanto en el JBC como en distintos puntos de la ciudad e incorporando además en su discurso, ideas claves de otros jardines de la RNJB, logrando alcanzar una dimensión nacional subjetiva interinstitucional.

II. Para el 2017 se asumieron posturas vinculantes con entidades tanto públicas como privadas retomando experiencias de los demás JB a nivel nacional, convirtiéndose el JBC en el octavo JB de Colombia y del mundo en realizar estos procesos, ni antes ni después de los acuerdos de paz que se han realizado en distintos Estados. Se sabe que los desmovilizados en los distintos países, sí se han incorporado a procesos como guardabosques o guardas forestales, pero ninguno en algún JB, siendo Colombia pionera en estas formas de reintegración a la vida civil a nivel social, de derechos humanos y de protección de la paz. 
Siendo actualmente el primer país en considerar interinstitucionalmente que todos los Jardines Botánicos (JJBB) de su territorio son Territorios de Paz.

III. El proceso del 2018, pasó de lo local a lo comunitario, lo institucional fue dentro de las instalaciones del JBC, lo local en algunos jardines, parques y la ribera del río Cali y lo comunitario con procesos que se generaron entre el JBC y el Zoológico de Cali, basados en la gestión social y en la creación de entornos paisajísticos de comunidades vulnerables que buscan embellecer el territorio y con ello generar un ambiente más limpio y auto sostenible como se ejecutó en el Barrio la Fortuna.

IV. Estos procesos se han basado bajo un pensamiento lógico simple, "si un JB tiene muchas oportunidades y necesidades de trabajo, esa necesidad y esas oportunidades, mezcladas con la necesidad de que estas personas cumplan unas horas, complementan las dos instituciones que son la ARN y el JBC" (E02), por tanto, el vínculo entre la ARN y demás instituciones, genera un resultado en el que las tres partes ganan, el JBC se beneficia de fuerza laboral horas voluntariado, las PPR realizan su Ruta a la vida civil generando un aporte a la comunidad y las instituciones que se vinculan cumplen con sus objetivos propuestos, generándose todo un beneficio en común y al mismo tiempo un aporte significativo en términos sociales y culturales. Sin embargo, la diferencia de este voluntariado es que también se pensó en el fortalecimiento y desarrollo de habilidades técnicas en las PPR, y en su formación ciudadana como agentes de cambios positivos en la sociedad.

V. Se hace evidente la posibilidad de un proyecto de gran envergadura, bien sea que se realice por medio del JBC, de manera regional con otros JJBB o con todos los JJBB a nivel nacional por medio de la RNJB, dado que los JJBB cuentan "con los recursos humanos para poder conceptualizar un proyecto que sea viable y que pueda trabajar de la mano con otras instituciones a nivel nacional e internacional para ejecutar proyectos que estén destinados a procesos socio ambientales" (E03).

VI. Existen dos aspectos importantes que se pueden resaltar en estos proyectos: 1) Las PPR se sienten útiles, "de poder venir y contribuir a dejar un legado a la comunidad en restauración ecológica, restauración de servicios, creación de espacios para la ciudadanía" (E03) y 2) En el sentido institucional, "tener personas que hayan o han estado inmersos en temas dolorosos para ellos mismos y para el país, nos permite contribuir a la reconciliación a 
través de generar estos espacios" (E03), por tanto, se abre un camino muy interesante y positivo hacia el futuro desde un enfoque científico, investigativo y de cambio social, resignificando de esta manera, estos espacios como los JJBB, en plataformas de construcción de ciudadanía y cultura de paz, con acciones visibles y de impacto en los proyectos de vida de quienes hacen parte del proceso.

VII. Se ha demostrado que la labor social por medio de trabajo voluntario genera riqueza económica, moral, social, psicológica, cultural y da las pautas necesarias para la reconciliación y la vuelta a la vida civil. Entonces es necesario pensar que estos procesos de restauración son primordiales, porque garantizan que los servicios ecosistémicos permanezcan en el tiempo, y eso hace que la reconciliación por parte de las PPR genere una conexión que realmente aporta a los procesos de olvidar, de no quedarse en los mismos dilemas que siempre han tenido los colombianos frente al CA y encaminarse hacia un país que construya su futuro a partir de la sinergia de todas las fuerzas.

VIII. La PPR, al salir de estos grupos, se dan cuenta que hay otra manera de vivir más acorde a lo social, que hay una normalidad, que tienen derechos civiles, políticos, sociales y culturales, con los cuales pueden realmente mejorar sus condiciones de vida tanto psicológica como material, dado que son personas que quieren cambiar, reinsertarse a la vida civil, con las cuales se puede laborar ya que son organizadas, disciplinadas, atentas y voluntariosas. Por tanto, todo el trabajo realizado de labor social en el JBC ha concedido a cada PPR hacer acciones de reparación, permitiendo que por sí mismas se concienticen y sienta que sus acciones y decisiones permiten retribuir al daño generado, que su reintegración genera una nueva forma de ver la vida, que existen segundas oportunidades y que de la guerra se puede pasar a la paz si hay voluntad y garantías sociales.

\section{Referencias bibliográficas}

Achutegui, P. (2017). Victimización de los delitos de odio. Aproximación a sus consecuencias

y a las respuestas institucional y social. Revista de Victimología, (5), 33-62. http://www.huygens.es/journals/index.php/revista-de-victimologia/article/view/82

Agencia Colombiaa para la Reincorporacion y la Normalizacion. ARN. (24 de febrero de 2017). Reseña Histórica Institucional. https://bit.ly/3s6vZiW

Agencia Colombiana para la Reincorporación y la Normalización. ARN. (22 de noviembre de 2019). Glosario ARN. https://bit.ly/3wFRWJj 
Agencia Colombiana para la Reincorporación y la Normalización. ARN. (9 de octubre de 2020). Histórico de personas desmovilizadas. https://bit.ly/3wM4hM6

Caicedo, L. P. (2016). Recomendaciones para un proceso de Desarme, Desmovilizacion y reintegracion $(D D R)$. https://bit.ly/3o2vWEF

Cervera, A. (2019). Cómo elaborar trabajos académicos y cientificos. Madrid: Alianza.

Consejo Nacional de Política Económica y Social. Conpes 3554. (1 de diciembre de 2008). Política nacional para la reintegración social y económica para personas y grupos armados ilegales. https://bit.ly/3fYXJs8

Consejo Nacional de Política Económica y Social. Conpes 3931. (22 de junio de 2018). Política nacional para la reincorporación social y económica de exintegrantes de las FARC-EP. https://bit.ly/3fY5LwI

Contreras, A. E. (2018). Desmovilizados de GAOML en procesos idseicos con Jardines Botánicos de Colombia. Grupos Armados Organizados al Margen de la Ley (GAOML) en los albores del tercer milenio (Trabajo maestría). Universitat Oberta de Catalunya, Barcelona, España. https://bit.ly/2OB3D2R

Diario Oficial No. 44.097. (24 de julio de 2000). Ley 599 de 2000. Código Penal. https://bit.ly/3t9pCgf

Human Rights Watch. (2003). Aprenderás a no llorar. https://bit.ly/2RpzKUb

Jardín Botánico de Cali. JBC. (2016a). Restaurando la vida: El Jardín Botánico de Cali como experiencia de Paz (power point). Recopilado. Isabel Elena Muñoz Galvis, Gloria Arboleda, Juan Sebastián Posada, Jaime Velásquez. Cali, Colombia: s.n., 2 de septiembre de 2016.

Jardín Botánico de Cali. JBC. (2016b). Propuesta De Servicio Social Agencia Colombiana Para La Reintegración. Recopilado. Gloria Arboleda, Cristian Guerrero Isabel Elena Muñoz Galvis. Cali, Colombia: s.n., 27 de abril de 2016.

Jardín Botánico de Cali. JBC. (2016c). Actividades Labor Social Agencia Colombiana De Reintegración. Recopilado. Isabel Elena Muñoz Galvis. Cali, Colombia: s.n., 19 de mayo de 2016.

Jardín Botánico de Cali. JBC. (2016d). Restaurando la vida: Resumen en Extenso. El Jardín Botánico de Cali como experiencia de Paz. Recopilado. Isabel Elena Muñoz Galvis, Gloria Arboleda, Juan Sebastián Posada, Jaime Velásquez. Cali, Colombia: s.n., 2 de septiembre de 2016. 
Jardín Botánico-Universidad Tecnológica de Pereira. UB-UTP. (2016). Encuentro de educación ambiental en jardines botánicos: Una apuesta para la paz. Pereira, Colombia. Documento Institucional. 18 de noviembre de 2016.

Kvale, S. (2011). Las entrevistas en investigación cualitativa. Madrid: Ediciones Morata

Laub, J. H., y Sampson, R. J. (2001). Understanding desistance from crime. Crime and justice, 28, 1-69.

Maruna, S. (2001). Making Good: How Ex-Convicts Reform and Rebuild their Lives. Washington, DC: American Psychological Association.

Naciones Unidas. NU. (2002). Protocolo facultativo de la Convención sobre los Derechos del Niño relativo a la participación de niños en los conflictos armados. https://bit.ly/324TEpm

Padrón, M. (2014). Expectativas de reinserción y desistimiento delictivo en personas que cumplen penas de prisión: factores y narrativas de cambio de vida (Trabajo de maestría). Universitat de Barcelona, Barcelona, España. https://bit.ly/2Qekc4R

SEMANA. (2009). Quince años en paz. https://bit.ly/3dOBLkr

UNICEF. (20 de junio de 2006). Convención de los Derechos del Niño de 1989. https://bit.ly/3dR22ON

\section{Entrevistas}

(E01) Entrevista a Isabel Elena Muñoz Galvis. Coordinadora de Educación del JBC. Cali. 02/09/2017.

(E02) Entrevista a Gloria Arboleda. Directora General JBC. Cali, 23/08/17.

(E03) Entrevista a Camilo José Londoño Andrade. Director Científico JBC. Cali, 29/08/18.

(E04) Entrevista a PPR. Jardinero JBC. Cali, 29/08/18.

(E05) Entrevista a PPR. Labor Social JBC, 22/08/17.

(E06) Entrevista a PPR. Labor Social JBC, 22/08/17.

(E07) Entrevista a PPR. Jardinero JBC, 22/08/17.

(E08) Entrevista a PPR. Jardinero JBC, 22/08/17.

(E09) Entrevista a PPR. Jardinero JBC, 23/08/18. 